\title{
Labeling Human Mesenchymal Stem Cells with Fluorescent Contrast Agents: the Biological Impact
}

\author{
Sophie E. Boddington, ${ }^{1}$ Elizabeth J. Sutton, ${ }^{1,2}$ Tobias D. Henning, ${ }^{1,3}$ \\ Alexander J. Nedopil, ${ }^{1}$ Barbara Sennino, ${ }^{4}$ Anne Kim, ${ }^{5}$ and Heike E. Daldrup-Link ${ }^{1}$ \\ ${ }^{1}$ Department of Radiology, University of California, San Francisco, San Francisco, CA, USA \\ ${ }^{2}$ Department of Radiology, Mt. Auburn Hospital, Boston, MA, USA \\ ${ }^{3}$ Department of Radiology, Technical University of Munich, Munich, Germany \\ ${ }^{4}$ Department of Anatomy, University of California, San Francisco, San Francisco, CA, USA \\ ${ }^{5}$ Department of Orthopedic Surgery, University of California, San Francisco, San Francisco, CA, USA
}

\begin{abstract}
Purpose: This study aims to determine the effect of human mesenchymal stem cell (hMSC) labeling with the fluorescent dye DiD and the iron oxide nanoparticle ferucarbotran on chondrogenesis.

Procedures: hMSCs were labeled with $\mathrm{DiD}$ alone or with $\mathrm{DiD}$ and ferucarbotran (DiD/ ferucarbotran). hMSCs underwent confocal microscopy, optical imaging (OI), and magnetic resonance (MR) imaging. Chondrogenesis was induced by transforming growth factor-b and confirmed by histopathology and glycosaminoglycan (GAG) production. Data of labeled and unlabeled hMSCs were compared with a $t$ test.

Results: Cellular uptake of DiD and ferucarbotran was confirmed with confocal microscopy. DiD labeling caused a significant fluorescence on $\mathrm{OI}$, and ferucarbotran labeling caused a significant T2* effect on MR images. Compared to nonlabeled controls, progenies of labeled MSCs exhibited similar chondrocyte morphology after chondrogenic differentiation, but the labeled cells demonstrated significantly reduced GAG production $(p<0.05)$.

Conclusion: DiD and DiD/ferucarbotran labeling of hMSC does not interfere with cell viability or morphologic differentiation into chondrocytes, but labeled cells exhibit significantly less GAG production compared to unlabeled cells.
\end{abstract}

Key words: Optical imaging, Magnetic resonance imaging, Molecular imaging, Cell labeling, Mesenchymal stem cell, Superparamagnetic iron oxide, DiD, Differentiation, Contrast agent

\section{Introduction}

$\mathrm{C}$ artilage lacks self-regeneration capacity, which makes -
regenerating structural damage to articular cartilage a particularly challenging problem. Stem cell transplants

Correspondence to: Sophie E. Boddington; e-mail: Sophie.Boddington@ radiology.ucsf.edu provide a potentially curative therapeutic option. However, there are many obstacles related to stem cell-mediated cartilage regeneration such as (a) achieving successful stem cell retention at the implantation site, (b) differentiation of the transplanted stem cells into chondrocytes, and (c) regeneration of hyaline cartilage instead of fibrous cartilage [1].

A noninvasive imaging technique for long-term in vivo detection of transplanted stem cells would significantly enhance our ability to identify factors that lead to cartilage 
regeneration and functional improvement of arthritic joints. Classical methods to monitor stem cell transplants have relied on postmortem histopathological analyses, which would not be clinically applicable. Novel noninvasive multimodality imaging approaches would be readily clinically applicable and could combine high sensitivity (as provided by optical imaging techniques) with direct cartilage depiction (as provided by magnetic resonance (MR) imaging) for high-resolution stem cell tracking in real time [2].

In order to track stem cells and/or their progenies in cartilage defects with a combined OI and MR imaging approach, the cells have to be labeled with markers that can be detected with either technique. Exogenous markers have the advantage of being easy to apply, while not being associated with genetic modifications of the labeled cells. However, it has been recently discussed, controversially, if cellular contrast agent markers may inhibit chondrogenesis of human mesenchymal stem cells (hMSCs) [3, 4]. Thus, the purpose of this study was to investigate the effect of hMSC labeling with the fluorescent dye DiD and the iron oxide nanoparticle ferucarbotran on chondrogenesis.

\section{Materials and Methods}

\section{Cell Culture}

After institutional approval and donor consent, primary hMSCs were aspirated from bone marrow (BM) in the iliac crest of a 20 year-old male patient with no known bone marrow pathology, who was admitted to our institution for trauma surgery. BM cells were plated and incubated for $12 \mathrm{~h}$. Nonadherent cells were removed and remaining cells were cultured in Dulbecco's modified Eagle medium (DMEM) high glucose media (Invitrogen, Carlsbad, CA, USA) supplemented with $10 \%$ fetal bovine serum (FBS; Hyclone, Logan, UT, USA) and $1 \%$ penicillin-streptomycin at $37^{\circ} \mathrm{C}$ in a humidified $5 \% \mathrm{CO}_{2}$ atmosphere. The culture media were changed every third day. The cells were passaged upon reaching $90 \%$ confluency and either redistributed to new culture flasks or used for experiments. All experiments were performed between passages 8-12 to optimize chondrogenic potential and avoid senescence. Three groups were investigated: nonlabeled hMSCs [1], hMSCs labeled with the fluorescent dye DiD [2], and hMSCs labeled with the MR contrast agent ferucarbotran in addition to the fluorescent dye DiD (DiD/ferucarbotran) [3].

\section{Cell Labeling Procedure}

1. $\mathrm{DiD}\left(\mathrm{C}_{67} \mathrm{H}_{103} \mathrm{CIN}_{2} \mathrm{O}_{3} \mathrm{~S}\right.$ : vibrant cell labeling solution, Molecular Probes, Eugene, OR, USA) is a lipophilic, carbocyanine near-infrared (NIR) fluorochrome with a molecular weight of 1,052 DA and excitation/ emission maximum of $644 / 665 \mathrm{~nm}$. Samples of $1.0 \times 10^{6} \mathrm{hMSC}$ were incubated with $5 \mu \mathrm{l}$ of DiD in $1 \mathrm{ml}$ of serum-free media for $20 \mathrm{~min}$ and then washed three times with phosphate-buffered saline (PBS) by sedimentation.

2. Ferucarbotran (Resovist: Bayer Schering AG, Berlin, Germany) consists of carboxydextran-coated superparamagnetic particles of iron oxides
(SPIOs). Ferucarbotran is characterized by a mean diameter of $60 \mathrm{~nm}$, a net negative charge, as well as $\mathrm{r} 1$ and $\mathrm{r} 2$ relaxivities of 25 and $151 \mathrm{mM}^{-1}$ $\mathrm{s}^{-1}$, respectively [5]. Samples of $1.0 \times 10^{6} \mathrm{hMSC}$ were incubated with ferucarbotran $(100 \mu \mathrm{g} \mathrm{Fe} / \mathrm{ml}$ serum-free high-glucose DMEM) for $2 \mathrm{~h}$. Then, 10\% FBS was added and cells were incubated for an additional $12 \mathrm{~h}$. The cells were carefully washed three times with PBS (pH7.4) by sedimentation $\left(5 \mathrm{~min}, 400 \mathrm{rcf}, 25^{\circ} \mathrm{C}\right)$. The ferucarbotran-labeled cell pellet was resuspended and labeled with DiD as described above. A serial dilution was prepared of $\mathrm{DiD} /$ ferucarbotran-labeled hMSCs and nonlabeled controls with decreasing cell numbers for subsequent MR and OI. Following each labeling procedure, the cells were counted using a hemocytometer (Neugebauer Chamber), and cell viability was tested by the trypan blue exclusion assay (Sigma Aldrich, St. Louis, MO, USA). Representative samples of labeled hMSC and unlabeled controls, prior to differentiation, were imaged using a Zeiss-LSM 510 confocal microscope (Carl Zeiss MicroImaging, Inc., Thornwood, NY, USA) to confirm labeling and localization. All hMSC samples were mounted with Vectashield containing 4',6-diamidino-2-phenylindole (DAPI; Vector Laboratories, Burlingame, CA, USA). Ferucarbotran-labeled samples were also stained with antidextran fluorescein isothiocyanate (FITC; Stem Cell Technologies, Tukwila, WA, USA) for iron localization.

\section{$M R I$}

In vitro imaging studies of hMSCs and chondrogenic cell pellets were performed with a 1.5-T MR scanner (Signa EXCITE HD; GE Medical Systems, Milwaukee, WI, USA). The chondrogenic pellets were imaged in polypropylene tubes and were immersed in a water bath $\left(20^{\circ} \mathrm{C}\right)$ to diminish susceptibility artifacts. The samples were scanned with a circularly polarized quadrature knee coil (Clinical MR Solutions, Brookfield, WI, USA). Axial sequences were obtained using a T2*-weighted GE sequence (TR 500, multiple TE 28.8:14.4:7.2:3.7 ms, BW 15.63 Hz, FOV12, matrix 256×196, NEX 1, alpha 30).

\section{$O I$}

Images of hMSCs and chondrogenic pellets were acquired and analyzed using Living Image 2.5 software (Xenogen, Alameda, CA, USA) integrated with Igorpro (Wavemetrics, Lake Oswego, OR, USA). The optical imager is an integrated fluorescence system $(400-900 \mathrm{~nm})$ that is composed of a light-tight specimen chamber (dark box) and a 0.5-in. charge-coupled device (CCD) camera. To minimize electronic background and maximize sensitivity, the CCD camera is thermoelectrically cooled to $-70^{\circ} \mathrm{C}$. Prior to acquiring the fluorescence-based optical images, gray scale images (i.e., a regular photograph) were acquired using unfiltered light from the excitation source, which were later fused with fluorescence images using IVIS software. All in vitro samples underwent OI using Cy5.5 filter preset (excitation filter passband 615-665 nm, emission filter passband $695-770 \mathrm{~nm}$, background filter passband $580-610 \mathrm{~nm}$ ) to match the absorption and emission characteristics of the labeling fluorophore, and unfiltered light from the excitation source was used for the photograph. Specifically, while DiD exhibits its maximum emission intensity at about $665 \mathrm{~nm}$, the emission spectrum extends to about $800 \mathrm{~nm}$ with about half of the emitted photon flux at wavelengths longer than $695 \mathrm{~nm}$, which allows them to be captured by the imaging system using the Cy5.5 filter set. The IVIS system has implemented a subtraction method to minimize the effect of residual background signal and autofluorescence that may be detected during fluorescent imaging. This subtraction method is executed by first acquiring an image with 
the primary excitation filter, which is then followed by acquiring another image with the background excitation filter. The background noise can then be subtracted. A detailed description of the imaging system is provided by Troy et al. [5]. Consistent illumination parameters were used for all NIR fluorescent acquisitions. Normalized images are obtained by dividing the fluorescent image by a reference illumination image, and thus, images are unitless. The resulting "normalized" image displays each pixel in the unit of "average efficiency" which represents the fractional ratio of fluorescent emitted photons per incident excitation photon (Xenogen, Alameda, CA, USA). For in vitro image analysis, regions-of-interest were defined as the circular area of the pellet.

\section{Chondrogenic Differentiation}

For each experimental group, four samples of $2.5 \times 10^{5} \mathrm{hMSCs}(n=4$ $\mathrm{DiD}, n=4 \mathrm{DiD} /$ ferucarbotran, and $n=4$ unlabeled control hMSC) were suspended in $0.5 \mathrm{ml}$ of complete chondrogenic media (Chondrogenic Differentiation Bulletkit; Lonza Group Ltd., Switzerland) prepared per manufacturer's protocol (Lonza Group Ltd., Switzerland) containing $10 \mathrm{ng} / \mathrm{ml}$ recombinant transforming growth factor (TGF)- $\beta 3$. The aliquot was transferred to $15 \mathrm{ml}$ polypropylene culture tubes (VWR, West Chester, PA, USA) and centrifuged at $150 \mathrm{rcf}$ for $5 \mathrm{~min}$ to form a pelleted micromass and subsequently incubated at $37^{\circ} \mathrm{C}$ in a humidified atmosphere of $5 \% \mathrm{CO}_{2}$. The media were changed every 2 days and the culture ended after 14 days.

\section{Glycosaminoglycan Quantification}

The absorbant assay, dimethylmethylene blue (DMMB), was used to assess the total glycosaminoglycan (GAG) content in the differentiation samples [6]. After the 14-day culture, each pellet was suspended in $0.5 \mathrm{ml} 1 \times \mathrm{TE}$ buffer, and the corresponding cumulative chondrogenic induction medium was homogenized for comparison to standard curves of chondroitin sulfate following the addition of $250 \mu \mathrm{l}$ DMMB solution (21 mg DMMB, $5 \mathrm{ml}$ absolute ethanol, and $2 \mathrm{~g}$ sodium formate; $\mathrm{pH} 3.5$ ). Average total GAG content and relative GAG content were calculated for each triplicate sample.

\section{Histopathologic Evaluation of Chondrogenic Differentiation}

The chondrogenic pellets were removed from test tubes, placed in $10 \%$ neutral buffered formalin, and then encapsulated in HistoGel (both Richard-Allan Scientific, Kalamazoo, MI, USA). The specimens were paraffin-embedded, sectioned into $5-\mu \mathrm{m}$ transverse slices, and stained with Alcian blue (stains acid mucopolysaccharides and glycosaminoglycans) and safranin-O (glycosaminoglycans).

\section{Statistical Analysis}

Imaging data and GAG data were displayed as means and standard deviations (SD). Data of labeled and unlabeled cells were tested for significant differences with a Student's $t$ test. Statistical significance was assigned for $p$ values $<0.05$.

\section{Results}

\section{Cell Labeling Procedure}

1. hMSC labeling with DiD: The trypan blue test revealed a cell viability of $97 \% \pm 3 \%$ before and $96 \% \pm 3 \%$ after the labeling procedure, which was not significantly different $(p>0.05)$. Confocal microscopy demonstrated that unlabeled hMSC showed no fluorescence (Fig. 1a) while there was uptake of DiD within the hMSC (Fig. 1b).

2. hMSC labeling with DiD/ferucarbotran: The trypan blue test revealed a cell viability of $96 \% \pm 3 \%$ before and $95 \% \pm 3 \%$ after the labeling procedure, which is not significantly different $(p>0.05)$. Confocal microscopy demonstrated the internalization of ferucarbotran in the cytoplasm of the cell (Fig. 1c).
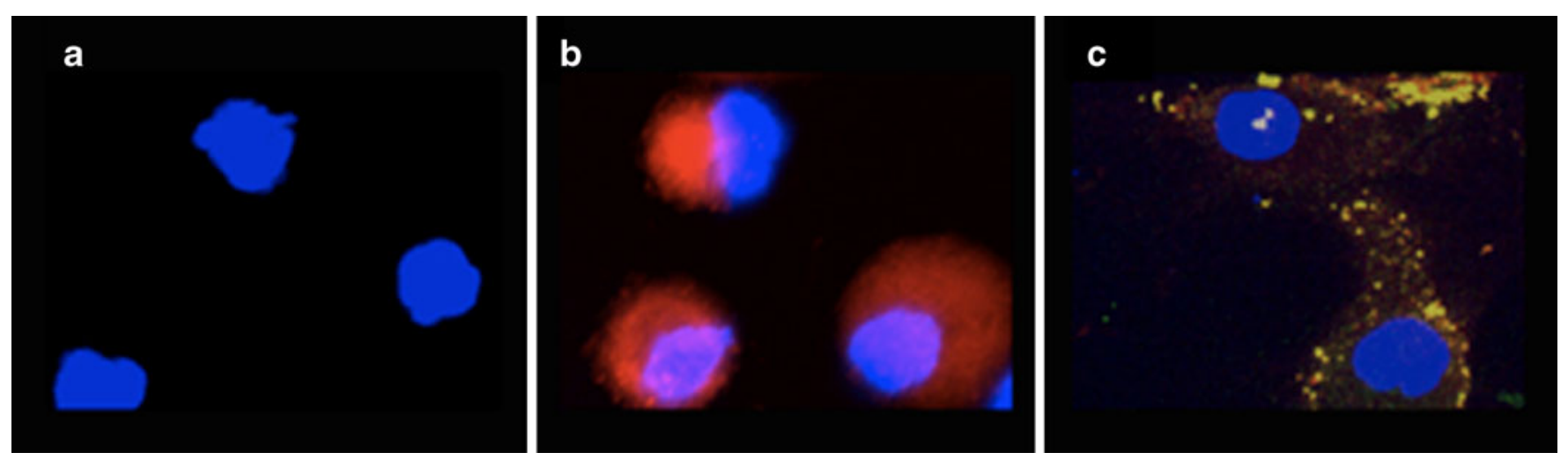

Fig. 1. High-power $(\times 40)$ confocal microscopy of a unlabeled control, b DiD-labeled hMSCs, and c DiD/ferucarbotran-labeled hMSCs stained with antidextran FITC stain. The nuclei of all hMSC have been counterstained with DAPI (blue channel). There was significant uptake of both contrast agents within the hMSC (b, c). 
a.

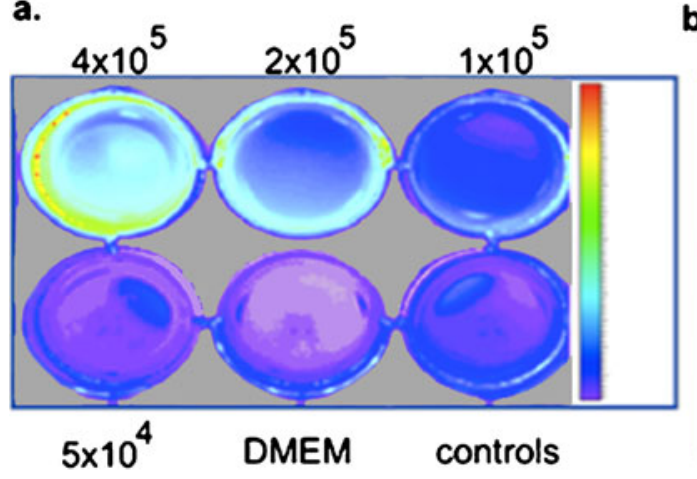

b.

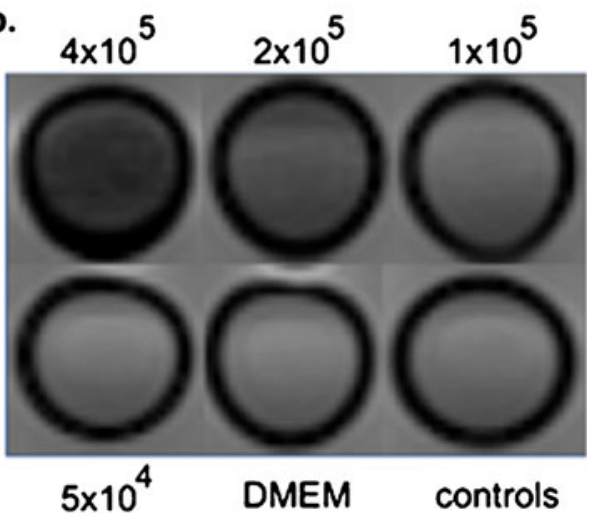

Fig. 2. A serial dilution was prepared of DiD/ferucarbotran-labeled hMSCs and unlabeled controls with decreasing cell numbers. a Ol signal of DiD/ferucarbotran-labeled hMSCs (units in efficiency) and nonlabeled controls showed increased fluorescent signal with increased cell numbers. b MR images of DiD/ferucarbotran-labeled hMSCs demonstrated a significantly decreased $\mathrm{T}^{*}$-signal with increasing cell number.

\section{Optical Imaging and MR Imaging}

1. Unlabeled hMSC did not show a significant fluorescent signal on OI images and showed a signal similar to culture medium on T2-weighted MR images immediately following labeling (Fig. 2a, b).

2. DiD-labeled hMSC showed a significantly stronger fluorescence with OI compared to unlabeled controls $(p<0.05$; Fig. 3a, b). The unlabeled control was used as the baseline sample and was normalized to zero. A fluorescent signal remained detectable throughout the differentiation culture, up to 14 days (Fig. 3b). The mean total fluorescent intensity of the DiD-labeled hMSCs (measured in units of efficiency) was $4.51 \mathrm{E}-07$ with a standard deviation of $2.19 \mathrm{E}-05$. The T2*-signal on MR images of DiD-labeled hMSC and unlabeled controls was not significantly different ( $p>0.05$; Fig. 3a, b).

3. DiD/ferucarbotran-labeled hMSC also showed a significantly stronger fluorescence with OI compared to unlabeled controls immediately following labeling (Fig. 2a), and this remained detectable following differentiation for up to 14 days (Fig. 3c). The unlabeled control was used as the baseline sample and was normalized to zero. The mean total fluorescent intensity of the DiD/ferucarbotran-labeled hMSC (measured in units of efficiency) following differentiation was $1.25 \mathrm{E}-06$ with a standard deviation of $6.13 \mathrm{E}-05$. In addition, $\mathrm{DiD} /$ ferucarbotran-labeled cells demonstrated a significantly decreased $\mathrm{T} 2 *$-signal compared to unlabeled controls on MR images (Figs. $2 \mathrm{~b}$ and $3 \mathrm{a}, \mathrm{c}$ ).

\section{Chondrogenic Differentiation}

1. Histologic analysis of the unlabeled cell pellets at 14 days after TGF-b induced successful differentiation supported by positive stains for Alcian blue and safranin-O (Fig. 4).
The average total GAG content of the unlabeled control was 23.31 (SD 6.30; Fig. 5).

2. The DiD-labeled pellet also revealed cells with similar architecture to that of the control supported by positive stains for Alcian blue and safranin-O (Fig. 4; Table 1). The average total GAG content of the DiD-labeled sample was 14.20 (SD 2.33). The relative GAG content

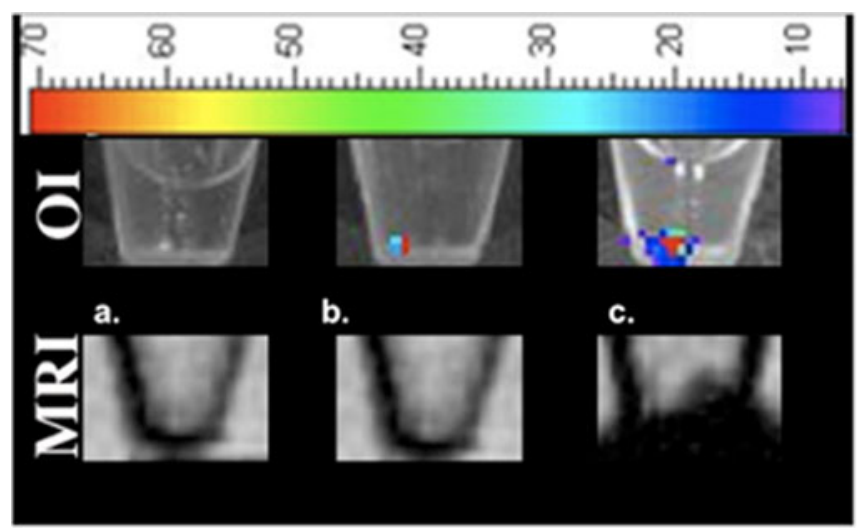

Fig. 3. Optical images (upper row) and MR images (lower row) of a, nonlabeled control pellet, $\mathbf{b}$ DiD-labeled pellet, and c DiD/ferucarbotran-labeled pellet. A representative color scale is provided which displays the fluorescence intensity above a certain threshold. All labeled pellets demonstrate a significant fluorescent signal on OI for the given threshold (b) c). The fluorescence signal is not homogenously distributed on the optical images due to the fact that the cells were centrifuged prior to imaging which caused the pellet to stick to one side. T2*-weighted MR images of DiD/ferucarbotranlabeled pellets demonstrate a significant decrease in signal (c). The MR signal seen in the lower row of $\mathbf{c}$ shows a blooming effect compared to the corresponding $\mathrm{Ol}$, which is caused by an extended range susceptibility effect of the magnetic particles within the cells. 

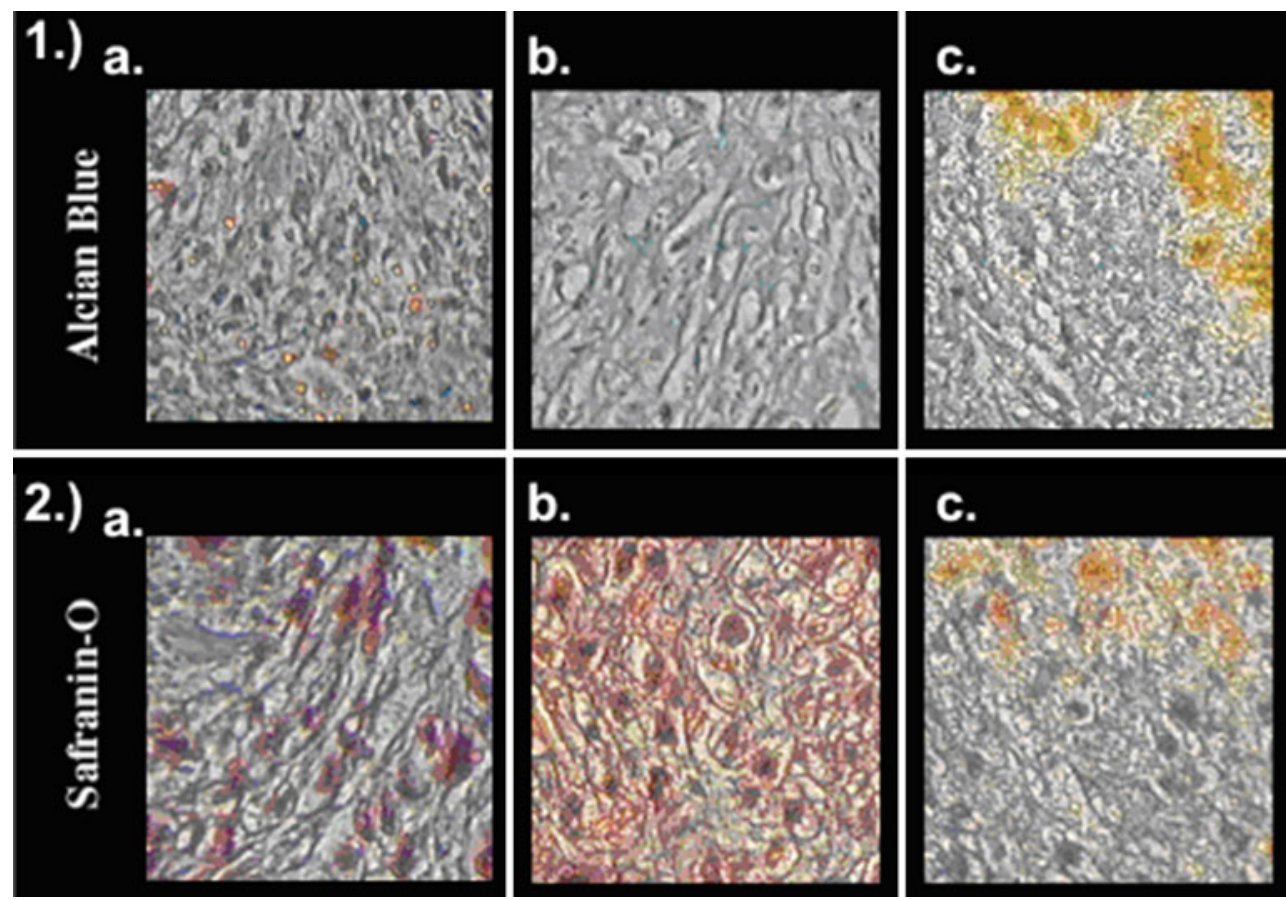

Fig. 4. Alcian blue stains of chondrogenic pellets: $1 a$ unlabeled control, $1 b$ DiD labeled, and 1c DiD/ferucarbotran labeled. $1 a-c$ Stained positive (blue stain of the intracellular and extracellular matrix). Safranin-O stains of chondrogenic pellets: $2 a$ unlabeled control, $2 b \mathrm{DiD}$ labeled, and $2 c$ DiD/ferucarbotran labeled. Chondrocytes are visible in all samples with the morphology of $2 b$ and $2 c$ similar to control. Iron can be visualized in $1 c$ and $2 c$.

of the DiD-labeled sample compared to the unlabeled control was 0.54 (SD 0.09). Total and relative GAG production of DiD-labeled hMSC was significantly reduced compared to unlabeled controls ( $p<0.05$; Fig. 5).

3. DiD/ferucarbotran-labeled samples showed similar chondrogenic differentiation to that of the control with positive stains for Alcian blue and safranin-O (Fig. 4; Table 1). The average total GAG content of the DiD/ferucarbotranlabeled pellets was 17.53 (SD 2.91). The relative GAG content was 0.75 (SD 0.12; Fig. 5). Compared to the control, DiD/ferucarbotran-labeled hMSC exhibited a significant inhibition in GAG production $(p<0.05)$.

\section{Discussion}

Our study demonstrates that hMSCs can be effectively labeled by simple incubation with $\mathrm{DiD}$ and $\mathrm{DiD}$ /ferucarbotran for depiction with OI and MR, without impairing the cells viability or proliferation. However, while histopathological evaluation of both unlabeled and labeled cells demonstrated differentiation into chondrocytes of similar morphology, labeled cells exhibited significantly less GAG production. Thus, our data suggest that the applied fluorescent and iron oxide contrast agent label do not interfere with morphological cell characteristics but may impair cell function.
Our data are in accordance with studies by Bulte and colleagues and Henning and colleagues, who recently described a dose-dependent inhibition of chondrogenesis with the SPIO based iron oxide labels ferumoxides and ferucarbotran $[3,4]$.

\section{MSC Chondrogenic Differentiation of Fluorescent and Bifunctional Labels}

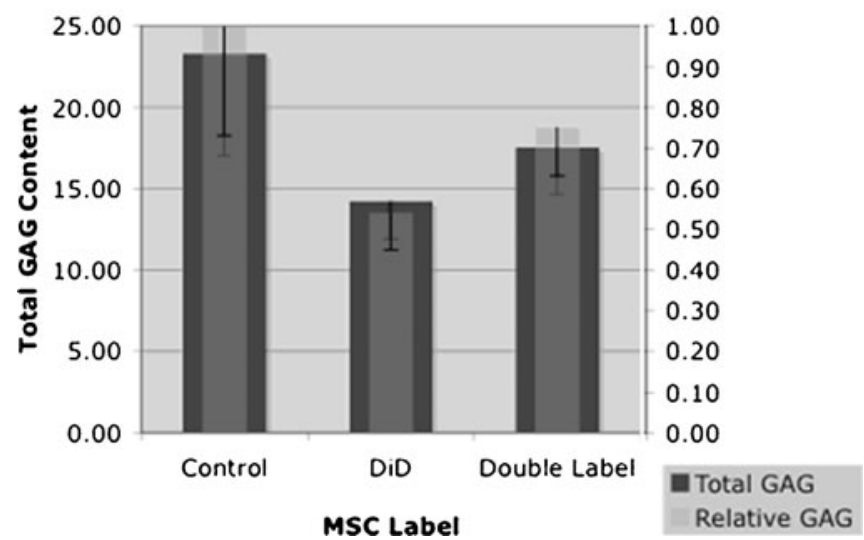

Fig. 5. Average total and relative GAG content of exogenously labeled chondrogenic pellets and controls: Data are displayed as means of triplicate samples with standard deviation. 
Table 1. Pathologic analysis of chondrogenic pellets

\begin{tabular}{llcclll}
\hline & Morphology & Differentiation & Safranin-O & Alcian blue & Iron content & Cell death \\
\cline { 2 - 6 } Control & Cartilage cells & 2 & Positive & Positive & None & None \\
DiD & Cartilage cells & 2 & Positive & Positive & None & None \\
Double level & Cartilage cells & 2 & Positive & Positive & Overloaded & Not significant
\end{tabular}

Pathologic analysis of chondrogenic pellets after staining with safranin-O and Alcian blue

0 no differentiation, 1 slight differentiation, 2 differentiation like control

Other authors reported a decline in cell proliferation after labeling neurons with Gd-based contrast agents [7], a diminished self-repair of mesenchymal stem cells after ferumoxides labeling [8] and a decreased function of natural killer cells after labeling with $\mathrm{FeCl} 3$ [9].

On the other hand, these same cells need iron for their metabolism and normal development $[10,11]$. Iron has a fundamental role in many metabolic processes, including electron transport, deoxyribonucleotide synthesis, oxygen transport, and many essential redox reactions involving hemoproteins [10]. Iron oxide nanoparticles are in fact appealing as contrast agents for cell labeling since two preparations are already FDA-approved (ferumoxides and ferumoxytol) and since their cellular uptake and intracellular metabolism have been extensively investigated in patients [12-14]. Likewise, labeling with fluorochromes has been reported to interfere with cell functions. For example, the fluorochrome calcein acetomethylester impaired the migration capacity of monocytes $[15,16]$. Conversely, other authors reported an unimpaired function of DiD-labeled natural killer cells [17].

A major challenge of cellular imaging is to develop optimized cell-labeling techniques, which provide a compromise between the minimal possible concentration for sensitive cell detection, and maximal possible concentration that does not impair cell function. Our data showed significantly less impairment in GAG production for chondrocytes labeled with $\mathrm{DiD} /$ ferucarbotran as opposed to cells labeled with DiD alone. A possible explanation for this observation may be a decreased uptake of DiD after prelabeling with ferucarbotran. These data support the theory of Bulte et al. that inhibition of chondrogenesis by contrast agent labeling is dose dependent [3] In addition, $\mathrm{DiD}$ integrates into the cell membrane and may interfere with the cell-cell contact needed for chondrogenesis [18].

We recognize several limitations to our study: Firstly, we examined stem cell differentiation in vitro; differentiation processes are more complex in vivo and have to be reevaluated in this context. Secondly, we focused our investigations on the fluorescent dye DiD and the iron oxide contrast agent ferucarbotran. Further studies have to investigate the effect of other fluorescent dyes and other MR contrast agents on stem cell differentiation. Lastly, we examined human mesenchymal stem cells; other stem cell types may be more or less sensitive to contrast agent labeling.

\section{Conclusions}

$\mathrm{DiD} /$ ferucarbotran labeling is appealing for monitoring stem cell transplants with "multimodality" imaging in order to integrate the high sensitivity provided by OI with the anatomical details provided by MR. Our data demonstrate that labeling with $\mathrm{DiD}$ and $\mathrm{DiD} /$ ferucarbotran enabled noninvasive long-term visualization of MSC and their progenies with OI and MRI but lead to inhibition of GAG production of labeled cells compared to controls. Thus, careful optimizations of labeling techniques are necessary in order to avoid interference with the cellular process that should be monitored.

Acknowledgements. This work was supported by a grant from the National Institute of Arthritis and Musculoskeletal and Skin Diseases (grant no. NIH R01AR054458).

Open Access. This article is distributed under the terms of the Creative Commons Attribution Noncommercial License which permits any noncommercial use, distribution, and reproduction in any medium, provided the original author(s) and source are credited.

\section{References}

1. Hunziker EB (1999) Articular cartilage repair: are the intrinsic biological constraints undermining this process insuperable? Osteoarthritis Cartilage 7:15-28

2. Liu W, Frank JA (2009) Detection and quantification of magnetically labeled cells by cellular MRI. Eur J Radiol 70:258-264

3. Kostura L, Kraitchman DL, Mackay AM, Pittenger MF, Bulte JW (2004) Feridex labeling of mesenchymal stem cells inhibits chondrogenesis but not adipogenesis or osteogenesis. NMR Biomed 17:513-517

4. Henning TD, Sutton EJ, Kim A et al (2009) The influence of ferucarbotran on the chondrogenesis of human mesenchymal stem cells. Contrast Media Mol Imaging 4:165-173

5. Troy T, Jekic-McMullen D, Sambucetti L, Rice B (2004) Quantitative comparison of the sensitivity of detection of fluorescent and bioluminescent reporters in animal models. Mol Imaging 3:9-23

6. Wolf F, Candrian C, Wendt D et al (2008) Cartilage tissue engineering using pre-aggregated human articular chondrocytes. Eur Cell Mater 16:92-99

7. Brekke C, Morgan SC, Lowe AS et al (2007) The in vitro effects of a bimodal contrast agent on cellular functions and relaxometry. NMR Biomed 20:77-89

8. Amsalem YMY, Daniels D, Ocherashvilli A, Holbova R, SharabaniYosef O, Feinberg MS, Barbash IM, Leor J (2006) Reparative capacity of mesenchymal stem cells is impaired by iron-oxide labeling after extensive myocardial infarction [abstract]. Circulation 114:661

9. Jiang XHK, Denicola D, Head JF, Elliott RL (2004) Iron impairs natural killer cell function [abstract]. In: AACR meeting

10. Shaw GC, Cope JJ, Li L et al (2006) Mitoferrin is essential for erythroid iron assimilation. Nature 440:96-100 
11. Richardson DR, Ponka P (1997) The molecular mechanisms of the metabolism and transport of iron in normal and neoplastic cells. Biochim Biophys Acta 1331:1-40

12. Wang YX, Hussain SM, Krestin GP (2001) Superparamagnetic iron oxide contrast agents: physicochemical characteristics and applications in MR imaging. Eur Radiol 11:2319-2331

13. Hyslop WB, Balci NC, Semelka RC (2005) Future horizons in MR imaging. Magn Reson Imaging Clin N Am 13:211-224

14. Corot C, Robert P, Idee JM, Port M (2006) Recent advances in iron oxide nanocrystal technology for medical imaging. Adv Drug Deliv Rev 58:1471-1504
15. Czepluch FS, Olieslagers SJ, Waltenberger J (2007) Monocyte function is severely impaired by the fluorochrome calcein acetomethylester. Biochem Biophys Res Commun 361:410-413

16. Szeto HH, Schiller PW, Zhao K, Luo G (2005) Fluorescent dyes alter intracellular targeting and function of cell-penetrating tetrapeptides. FASEB J 19:118-120

17. Tavri S, Jha P, Meier R et al (2009) Optical imaging of cellular immunotherapy against prostate cancer. Mol Imaging 8:15-26

18. Lee JW, Kim YH, Kim SH, Han SH, Hahn SB (2004) Chondrogenic differentiation of mesenchymal stem cells and its clinical applications. Yonsei Med J 45(Suppl):41-47 